\title{
MEMBRANE CHARACTERISTICS OF PALLADIUM-SAMARIUM ALLOY FOILS: MECHANICAL PROPERTIES AND HYDROGEN PERMEABILITY
}

\author{
${ }^{1}$ Natalia B. KOLCHUGINA, ${ }^{1}$ Semen V. GORBUNOV, ${ }^{1}$ Natalia R. ROSHAN, \\ ${ }^{1}$ Gennadii S. BURKHANOV, ${ }^{1}$ Nikolay A. DORMIDONTOV, ${ }^{1,2}$ Mark V. ZHELEZNYI, \\ ${ }^{1}$ Anna S. BAKULINA \\ ${ }^{1}$ Baikov Institute of Metallurgy and Materials Science, Russian Academy of Sciences, Moscow, Russia, \\ merciles@mail.ru,nkolchugina@imet.ec.ru \\ ${ }^{2}$ National University of Science and Technology MISiS, Moscow, Russia, markiron@mail.ru
}

https://doi.org/10.37904/metal.2020.3605

\begin{abstract}
Palladium membranes have a high selectivity for hydrogen, which is coupled with their temperature and mechanical stability. Such a selectivity of palladium membranes makes the membrane method the single procedure that allows one to prepare the high-purity hydrogen (no less than 99.9999 vol\%) being the main energy carrier in hydrogen power engineering. Palladium-rare earth metal solid solutions are possible hydrogen diffusion membrane alloys, since they exhibit the mechanical stability during the permeation along with the high hydrogen permeability characteristic. Palladium-samarium alloys of nominal compositions Pd2.6, 3.2, 5.2, 6.8, 8.3, 9.5, and 11 at\% Sm were prepared by arc melting; ingots were subjected to free forging and cold rolling to a thickness of $50 \mu \mathrm{m}$ with intermediate annealing. Vickers hardness, ultimate tensile strength, and relative elongation, were determined and the hydrogen permeability was measured. Within the solid-solution compositions, the strengthening takes place; the ultimate strength increases from $200 \mathrm{MPa}$ (for $\mathrm{Pd}$ ) to $830 \mathrm{MPa}$ for $\mathrm{Pd}-8.3 \mathrm{at} \% \mathrm{Sm}$. In this case, the relative elongation remains close to that of pure $\mathrm{Pd}(21$ $\%)$. Such mechanical characteristics favor the manufacturing ultrathin foils and show promise for using them in high-performance membranes and membrane elements. The alloys demonstrate the high hydrogen permeability at $300-600 \mathrm{~K}$, which is higher substantially as compared to that of Pd alloys alloyed with other elements. Variations of mechanical properties and hydrogen permeability are discussed from the viewpoint of the possible ordering of the solid solutions and formation of hydride phases.
\end{abstract}

Keywords: Palladium-samarium alloys, solid solution, foil, hydrogen permeability, mechanical properties

\section{INTRODUCTION}

Industrial application of palladium membranes can result in significant advance in the separation of hydrogen from gas mixtures and in the development of innovative applications of palladium alloys. Palladium membranes have a high selectivity for hydrogen, which is coupled with their temperature and mechanical stability. Such a selectivity of palladium membranes makes the membrane method the single procedure that allows one to prepare the high-purity hydrogen (no less than 99.9999 vol\%) being the main energy carrier in hydrogen power engineering.

The disadvantage of palladium membranes, which limits their large-scale application, consists in the medium hydrogen permeability and high cost. The lowering the palladium membrane thickness, still preserving the mechanical strength, would addressed the problems of membranes for the hydrogen separation. For this reason, the attention of investigators is focused on the search for components for the alloying of palladium, which allow one to increase its hydrogen permeability and to ensure the adequate mechanical strength and plasticity for manufacturing thin foils from the alloyed palladium-based compositions. 
It was shown [1] that palladium-rare earth metal (REM) solid solutions are possible hydrogen diffusion membrane alloys, especially because the alloys containing about 8 at\% $Y$ exhibit the mechanical stability during the permeation along with the high hydrogen permeability characteristic. The composition with 8 at $\%$ is the critical composition for the disappearance of the hydrogen miscibility gap in the $\mathrm{Pd}-\mathrm{Y}-\mathrm{H}$ system. The closure of the miscibility gap is one the main factors controlling the hydrogen solubility in palladium-REM alloys and takes place at an electron-to-atom (e/a) ratio of 0.24 .

The maximum solid solubility of REMs in palladium is anomalously high. The expansion of the palladium lattice due to the REM solubility is great [2]. Therefore, there is significant solid solution hardening in these alloys and, thus, the possibility of manufacturing a stronger membrane material.

Unlike the traditional $\mathrm{Pd}-\mathrm{Y}$ alloys that are well understood from the viewpoint of interaction with hydrogen, the Pd-Sm compositions were studied only in a few works, in particular, in [2] and [3].

According to the Pd-Sm phase diagram [4], the samarium solubility in palladium is 10.38 at\% at $1351 \mathrm{~K}$ (the eutectic temperature). As the temperature decreases, the $\mathrm{Sm}$ solubility decreases. The region of limiting $\mathrm{Sm}$ solubility in $\mathrm{Pd}-\mathrm{Sm}$ alloys corresponds to the short-range ordered $\mathrm{Pd}_{7} \mathrm{Sm}$ phase (12.5 at\% $\mathrm{Sm}$ ), which coexists with the fcc Sm solid solution in Pd (substitutional solid solutions with the Cu-type structure, space group Fm$3 m$ ). The formation of the ordered phase is accompanied by a peritectoid reaction: $\alpha-P d+\mathrm{Pd}_{5} \mathrm{Sm}(16.7 \mathrm{at} \%$ $\mathrm{Sm}$ ) at $547^{\circ} \mathrm{C}$ (in contrast to the other Pd-REM systems, in which the reaction $\alpha-\mathrm{Pd}+\mathrm{Pd}_{3} \mathrm{Sm}$ takes place).

The lattice parameter of palladium increases almost linearly with increasing Sm content [2]. The magnitude of the palladium lattice expansion due to addition of $\mathrm{Sm}, \Delta \mathrm{a}_{\mathrm{s} s} / \Delta \mathrm{x}_{\mathrm{u}}$ (where $\mathrm{x}_{\mathrm{u}}$ is the solute atomic fraction), which determines the hydrogen diffusion, is higher than that for $\mathrm{Y}$. However, contrary to expectation, the lattice expansion of the Pd-Sm is slightly smaller when compared with the tendency of the other rare earth metals and their atomic number [2].

The aim of the present work is to prepare Pd-Sm alloys with from 2.6 to 11 at\% Sm in the form of foils $50 \mu m$ thick and to study regularities of variations of their mechanical properties and hydrogen permeability at different temperatures.

\section{EXPERIMENTAL}

Palladium-samarium alloys of nominal compositions $\mathrm{Pd}-2.6,3.2,5.2,6.8,8.3,9.5$, and $11 \mathrm{at} \% \mathrm{Sm}$ were prepared by arc melting using $\mathrm{Pd}$ of $99.95 \mathrm{wt} \%$ purity and distilled samarium $99.9 \mathrm{wt} \%$ purity. The melting was performed in a purified argon atmosphere using a water-cooled bottom and nonconsumable tungsten electrode. The chemical compositions of alloys were determined by X-ray fluorescent analysis using a VRA33 spectrometer (Carl Zeiss, Germany).

After a homogenizing annealing at $1223 \mathrm{~K}$ for $5 \mathrm{~h}$ in a vacuum, the rectangular ingots were subjected to free forging and cold rolling to a thickness of $50 \mu \mathrm{m}$ (using Duo and Quarto mills) with an intermediate annealing. Finally, the foils were annealed at $1123 \mathrm{~K}$ for $1 \mathrm{~h}$ in a vacuum and subjected to furnace cooling.

The Vickers hardness of the foils was studied using a 401/402-MVD hardness tester (Instron Wolpert Wilson Instruments). The mechanical characteristics, ultimate tensile strength and relative elongation of the foils in the annealed state were determined using an Instron 3382 testing machine.

The hydrogen permeability was measured by filling a calibrated volume using an original experimental set up equipped with a high-temperature cell. The diameter of operating membrane surface is $20 \mathrm{~mm}$; the range of operating temperatures is $340-923 \mathrm{~K}$. The range of operating pressures before and after the membrane can vary from 0.1 to $2.5 \mathrm{MPa}$ and from 0.02 to $1.0 \mathrm{MPa}$, respectively.

Using results of measurements, the specific hydrogen permeability was calculated for a membrane $1 \mathrm{~mm}$ thick for each of measuring temperatures by the expression: 


$$
Q=\frac{\Delta P \cdot V_{\mathbf{k}} \cdot T_{\mathbf{0}} \cdot S}{T_{1} \cdot p_{0} \cdot F \cdot \tau\left(\sqrt{p_{e n t}}-\sqrt{p_{e x}}\right)}
$$

where

$V_{k}$ - the calibrated volume $\left(\mathrm{m}^{3}\right) ; \Delta p$ - the pressure difference in the calibrated volume when filling the sell with hydrogen (MPa); $T_{0}=273 \mathrm{~K} ; s$ - the membrane thickness $(\mathrm{mm}) ; T_{1}$ - the ambient temperature $(\mathrm{K})$; $p_{0}=0.1 \mathrm{MPa} ; S$ - the area of operating membrane surface $\left(\mathrm{m}^{2}\right) ; \tau$ - the time of calibrated volume filling (h); $p_{\text {ent }}$ - the pressure before the membrane (MPa); $p_{\text {ex }}$ - the pressure after membrane (MPa).

\section{RESULTS}

\subsection{Mechanical properties}

The hardness (Figure 1), ultimate tensile strength, and relative elongation (Figure 2) of $50 \mu \mathrm{m}$ foils were measured in accordance with their composition.

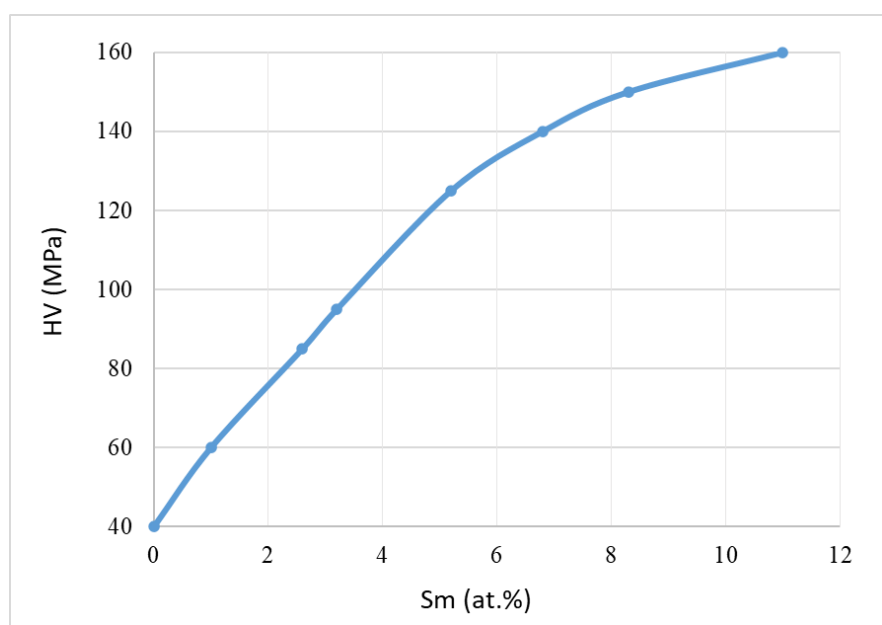

Figure 1 Composition dependence of the Vickers hardness of the Pd-Sm foils $50 \mu \mathrm{m}$ in thickness

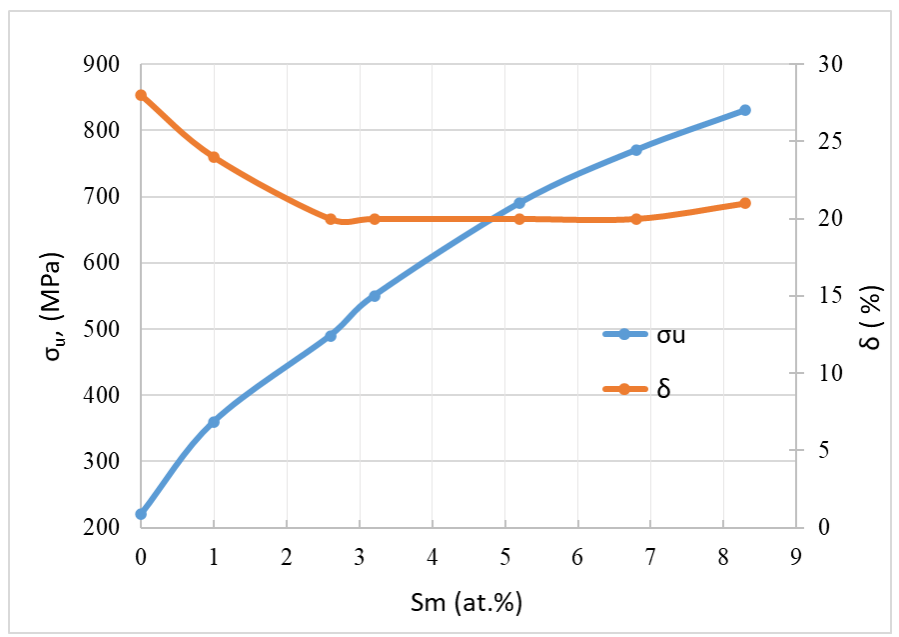

Figure 2 Ultimate tensile strength $\left(\sigma_{u}\right)$ and relative elongation $(\delta)$ of Pd-Sm foils $50 \mu \mathrm{m}$ in thickness

It is seen that the hardness of the foils increases monotonically from $400 \mathrm{MPa}$ (pure palladium) to $1600 \mathrm{MPa}$ for the Pd-11 at\% Sm composition. The ultimate strength $\sigma_{u}$ for palladium is $200 \mathrm{MPa}$. The Pd-8.3 at\% Sm foil 
exhibits an ultimate strength of $830 \mathrm{MPa}$; in this case, the relative elongation of Sm solid solutions is sufficiently high, namely, $\delta=21 \%$ for the Pd-8.3 at\% Sm composition.

The hardness measurements allow us to estimate the effect of Sm on the strengthening of palladium. As is known [5], the solid solution hardening is due to the atomic size misfit. The studied foils correspond to the fcc $\mathrm{Pd}$-based solid solution. The lattice parameter of the solution varies linearly with composition up to 8 at\% [2]. In [5], the common feature of the compositional dependences of the hardness of a number of REM solid solutions in palladium is a marked decrease in rate of the hardness increase with increasing the REM concentration, which is observed around the middle of the solid solution range.

The analysis of the compositional dependence of the hardness (Figure 1) of Pd-Sm alloys allows us to divide the curve into two portions. The first portion includes a range of 0 to $\sim 5$ at $\% \mathrm{Sm}$ and is characterized by the abrupt increase in the hardness. The composition with 5 at\% Sm corresponds to the middle of the solid solution range. The further increase in the Sm content results in the decrease in the increment in the hardness per atomic percentage of samarium. It should be noted that, according to the $\mathrm{Pd}$-Sm phase diagram [4], at room temperature, the Sm solubility in Pd does not exceed $\sim 4$ at\% Sm. At the higher Sm contents, the Sm solid solution is in equilibrium with the $\mathrm{Pd}_{7} \mathrm{Sm}$ compound.

It is obvious that the hardness variations are due to changes in the solid solution lattice, namely, the transition from a disordered to a short-range ordered (SRO) solid solution with increasing samarium content. The palladium-rare-earth solid solutions are ideal candidates for the appearance of SRO since there are very significant differences in both the atomic sizes and the electronegativities of the component atoms [5]. Since the $\mathrm{Sm}$ solid solution is in equilibrium with $\mathrm{Pd}_{7} \mathrm{Sm}$ compound, the $\mathrm{SRO}$ is assumed to be based on the $\mathrm{Pd} \mathrm{d}_{7} \mathrm{Sm}$ superlattice [4]. The variations of the hardness with increasing Sm content can be explained based on assumptions available in [5], namely, in terms of a rapid increase of the strain energy for more disordered solid solutions, and the subsequent relief of this increase in strain energy with composition by the appearance of extensive SRO at higher Sm contents.

According to [3], Pd-Sm alloys in the composition range between 5.0 and 15.0 at\% Sm indicate the presence of an ordered phase in the form of a $\mathrm{Pd}_{7} \mathrm{Sm}$ superlattice. However, the dependence of the ultimate tensile strength of $\mathrm{Pd}-\mathrm{Sm}$ alloys is characterized by monotonic increase in $\sigma_{u}$ with increasing $\mathrm{Sm}$ content and exhibits no anomalies. According to [6], the large strengthening effect is high for light rare-earth elements and is medium for $\mathrm{Sm}$; there is a linear relationship between the tensile strength and the rare-earth metal concentration which is valid for dilute concentration up to 0.6 at\% REM. According to the data, the increase in the tensile strength of the solid solution is $33 \mathrm{MPa}$ per $0.1 \mathrm{at} \% \mathrm{Sm}$. Our results agree with the literature data. The concentration dependence of the relative elongation exhibits first the decrease in $\delta$ up to $2.5 \% \mathrm{Sm}$ as compared to that of $\mathrm{Pd}$ and, after that, the relative elongation remains almost unchanged.

\subsection{Hydrogen permeability}

In the palladium-hydrogen system two fcc phases, designated $\alpha$ and $\beta$ with low and high concentrations of hydrogen, respectively, can co-exist at room temperature over a range of hydrogen contents ( $\alpha$ and $\beta$ which may be termed the $\alpha / \beta$ miscibility gap) [7]. The alloying with REMs suppresses the formation of $\beta$ phase and it was found that, under operating conditions, a continuous metal-hydrogen phase ( $\alpha$ ) could be guaranteed for palladium alloys containing 8 at\% REM $(6.5 \% \mathrm{Ce})$ [1].

Figures 3 and $\mathbf{4}$ show the temperature and composition dependences of the hydrogen permeability of Sm-Pd alloys, respectively.

The variations of the hydrogen permeability (Figure 3), which are characterized by a maximum and observed for the compositions with to $6.8 \mathrm{at} \% \mathrm{Sm}$, are related to the existence of $\alpha / \beta$ miscibility gap in the alloys. For the compositions with more than 8 at\% Sm, no maximum in the dependence is observed. 
As it was mentioned above, the hydrogen permeability is greatly increased by the presence of the short-range ordered phase [2]; the diffusivity of hydrogen in the ordered alloys is much greater than in the disordered state, but the ordered alloys dissolve less hydrogen than do the disordered alloys. In the case of Pd-Sm system, the ordered phase $\mathrm{Pd}_{7} \mathrm{Sm}$ appears in the compositions with more than $\sim 5$ at\% $\mathrm{Sm}$, and the hydrogen permeability of the alloy with 5.2 at\% begins to increase (Figure 4). Its increase becomes more substantial for the compositions with more than 8 at\% Sm. For the compositions with more than $10 \mathrm{at} \% \mathrm{Sm}$, the presence of the $\mathrm{Pd}_{5} \mathrm{Sm}$ ordered phase is possible [4].

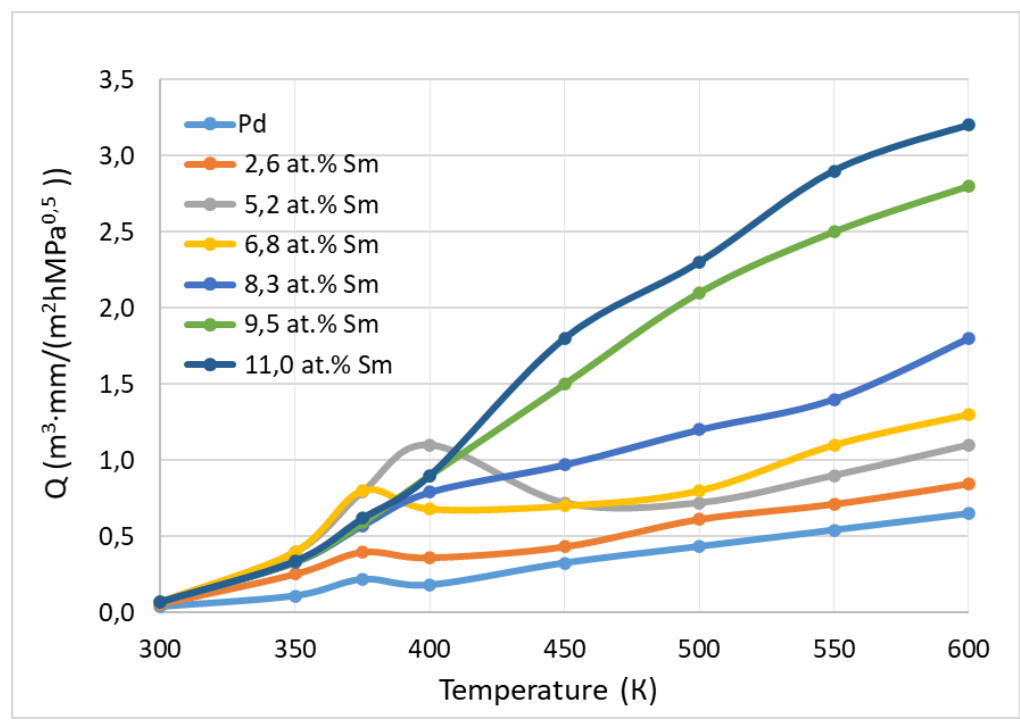

Figure 3 Temperature dependences of the hydrogen permeability of Pd-Sm foil alloys

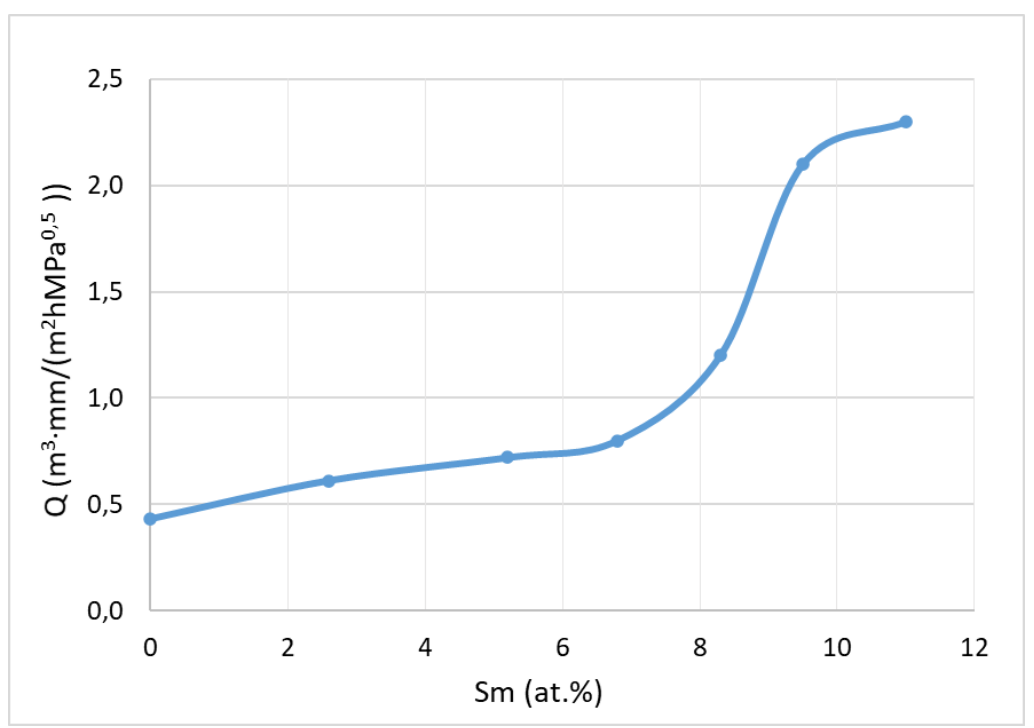

Figure 4 Composition dependence of the hydrogen permeability of $\mathrm{Pd}-\mathrm{Sm}$ foil alloys at $523 \mathrm{~K}$

The hydrogen permeability of Pd-Sm alloys at low temperatures (to $550 \mathrm{~K}$ ) is higher substantially than that of Pd (1.5-2 times).

It should be noted that, according to [2] Pd-Sm alloys seem to be better candidates for use as hydrogen purification membranes (as compared to Pd membranes) because these alloys have a larger lattice expansion at the critical composition (corresponding to the disappearance of the $\beta$ phase), and large lattice expansion is, in general, favorable for hydrogen diffusion. 


\section{CONCLUSION}

The Pd-Sm foils $50 \mu \mathrm{m}$ in thickness with Sm contents of 2.6, 3.2, 5.2, 6.8, 8.3, 9.5, and 11 at\% were prepared by cold rolling. The hardness, ultimate tensile strength, and relative elongation of the foil alloys were studied. The substantial solid solution strengthening was demonstrated; the relative elongation remains close to that of pure $\mathrm{Pd}(21 \%)$. Such mechanical characteristics favor the manufacturing ultrathin foils and show promise for using them in high-performance membranes and membrane elements.

The Pd-Sm alloys demonstrate the high hydrogen permeability at moderate temperatures (to $600 \mathrm{~K}$ ), which is higher substantially as compared to that of $\mathrm{Pd}$-based compositions alloyed with other elements. This fact determines the possibility of using the Pd-Sm membrane in performing low-temperature catalytic processes.

Variations of mechanical properties and hydrogen permeability are discussed from the viewpoint of the possible ordering of the solid solutions ( $\mathrm{Pd}_{7} \mathrm{Sm}$ superlattice) and formation of hydride phases.

\section{ACKNOWLEDGEMENTS}

This study was supported by the Ministry of Science and Higher Education of the Russian Federation, Agreement no. 05.604.21.0244, Unique identification number RFMEFI60419X0244.

\section{REFERENCES}

[1] DOYLE, M. L., HARRIS, I. R. Palladium-rare earth alloys: Their order-disorder transformations and behavior with hydrogen, Platinum Metals Review. 1988, vol. 32, pp. 130.

[2] CHEN, F.L., FURUKAWA, M., SAKAMOTO, Y. X-ray investigations of the $\alpha-\beta$ miscibility gaps in hydrogenated palladium-rare earth alloys. Journal of the Less-Common Metals. 1989, vol. 155, pp. 173-184.

[3] SAKAMOTO, Y., TAKAO, K., TAKEDA, S., TAKEDA, T. Evidence for an ordered Pd7Sm phase in Pd-Sm alloys. Journal of the Less-Common Metals. 1989, vol. 152, pp. 127-138, https://doi.org/10.1016/0022-5088(89)90077-5.

[4] OKAMOTO H. Pd-Sm (Palladium-Samarium). Journal of Phase Equilibria. 2001, vol. 22, no. 3, pp. $364-365$.

[5] HIRST, J. R., WISE, H M. L.., FORT, D., FARR, G J. P., HARRIS, I. R., Hydrogen softening in solid solution alloys. Journal of the Less-Common Metals.1976, vol. 49, pp. 193-211.

[6] NING, Y. Alloying and Strengthening Effects of Rare Earths in Palladium. Platinum Metals Review. 2002, vol.46, no. 3, pp. 106-115.

[7] MCNICHOLL, R.-A., LEWIS, F. A. The Hydride Phase Miscibility Gap in Palladium-Rare Earth Alloys, x-ray and equilibrium hydrogen pressure studies. Platinum Metals Review. 1990, vol. 34, no. 2, pp. 81-84. 